\title{
ESTUDIO DE LAS JORNADAS LABORALES DE 12 HORAS EN LA INDUSTRIA MANUFACTURERA
}

\author{
STUDY OF 12-HOUR WORKING DAYS IN THE MANUFACTURING INDUSTRY
}

\author{
Daniela Inzunza Robles* \\ Gilberto Orrantia Daniel $^{* *}$ \\ Enrique de la Vega Bustillos ${ }^{* * *}$ \\ German Alonso Ruiz Domínguez ${ }^{* * * *}$ \\ Rodolfo Ulises Rivera Landaverde $e^{* * * *}$
}

\begin{abstract}
Resumen: En el presente estudio se investigan los factores de riesgo de los turnos laborales de 12 horas, mediante un análisis de salud ocupacional, que incluye evaluación de fatiga, de riesgo ergonómico y factores de riesgo psicosocial, en una empresa manufacturera de la región, dedicada a la elaboración de arneses para la industria automotriz. La evaluación de fatiga se realiza con el cuestionario subjetivo de fatiga de Yoshitake, para los factores de riesgo psicosocial se emplea la Norma Oficial Mexicana NOM-035-STPS-2018 y, por último, la herramienta ART Tool, para evaluar factores de riesgo ergonómico. Los resultados del cuestionario de Yoshitake, arrojaron un incremento en el índice de queja de fatiga, entre el medio turno y al finalizar el mismo. Respecto a los factores de riesgo psicosocial se obtuvo un resultado global de riesgo bajo, en el análisis de cada línea de producción se obtuvieron que las líneas A y D presentaron un nivel de riesgo nulo, en las líneas B, E y $\mathrm{F}$ se obtuvo un nivel de riesgo bajo. La línea $\mathrm{C}$ obtuvo un nivel de riesgo medio, correspondientes a las categorías denominadas "factores propios de la actividad" y "organización del tiempo de trabajo", según la clasificación del cuestionario aplicado. Respecto a los factores de riesgo ergonómico, se encontró que $12,07 \%$ de las operaciones se encuentran con un nivel de riesgo bajo, 24,14\% corresponde a un nivel medio y el $63,78 \%$ corresponde a un nivel de riesgo alto.
\end{abstract}

Palabras clave: Fatiga, riesgo psicosocial, riesgo ergonómico, Yoshitake, estrés.

\begin{abstract}
In the present study, the risk factors of 12-hour work shifts are investigated, through an occupational health study, which includes assessment of fatigue, ergonomic risk and psychosocial risk factors, in a manufacturing company in the region, dedicated to the development of harnesses for the automotive industry. The evaluations of the study are carried out in the transmission area, with men and women for the analysis. The fatigue evaluation is carried out with the Yoshitake subjective fatigue
\end{abstract}

\footnotetext{
"Tecnológico Nacional de México/IT. Hermosillo, Sonora, México. Correo electrónico: danniela.inzunza@hotmail.com. Orcid: https://orcid.org/oooo-0oo1-9218-8212. Autor de correspondencia.

**Tecnológico Nacional de México/IT. Hermosillo, Sonora, México. Correo electrónico: orrantiadaniel@hotmail.com. Orcid: https://orcid.org/oooo-0002-7191-0656

${ }^{* * *}$ Tecnológico Nacional de México/IT. Hermosillo, Sonora, México. Correo electrónico: en_vega@ith.mx. Orcid: https://orcid.org/oooo-0002-0761-6858

****_Tecnológico Nacional de México/IT. Hermosillo, Sonora, México. Correo electrónico: gruiz@ith.mx. Orcid: https://orcid.org/oooo-0002-1497-5066

******Tecnológico Nacional de México/IT. Hermosillo, Sonora, México. Correo electrónico: ulisesriv@yahoo.com.mx. Orcid: https://orcid.org/oooo-0oo1-9230-2441
} 
questionnaire, for psychosocial risk factors the Official Mexican Standard NOM-035-STPS-2018 is used and, finally, the instrument called ART Tool, to evaluate risk factors ergonomic. The results of the Yoshitake questionnaire showed an increase in the fatigue complaint index between the half shift and at the end of the shift. For psychosocial risk factors, the following results were obtained. As a final global result of the survey, there is a low-risk level. Analyzing each production line, it was obtained that in lines $\mathrm{A}$ and $\mathrm{D}$, there is a null risk level, in lines $\mathrm{B}, \mathrm{E}$, and $\mathrm{F}$, a score was obtained that the places at a low-risk level. Finally, line $\mathrm{C}$ is at a medium risk level. On the other hand, when classifying this questionnaire's domains, the category called "Factors specific to the activity" and " Organization of working time "is in the medium-risk levels. For ergonomic risk factors, it was found that $12.07 \%$ of jobs have a low-risk level, $24.14 \%$ corresponds to a medium risk level, and $63.78 \%$ corresponds to a high-risk level. It is advisable to continue with risk factor evaluations for future interventions.

Keywords: Fatigue, psychosocial risk, ergonomic risk, Yoshitake, stress.

Recepción: 27.04.2021 / Revisión: 29.04.2021 / Aceptación: 06.05.2021

\section{Introducción}

La globalización, la economía mundial, la competitividad, la industria y la revolución informática, juegan un papel de primera instancia en la sociedad. Estos elementos clave, han dado la pauta a transformaciones profundas en las condiciones de vida, de trabajo y de salud, en la mayoría de los trabajadores mexicanos (Villegas et al., 1997).

Todos estos cambios, han aumentado la velocidad de los procesos de negocios y han establecido una sociedad de 24 horas. Debido a necesidades económicas, de producción o sociales, en algunos colectivos profesionales ha sido necesario establecer turnos de trabajo que permitan ofrecer una continuidad en los servicios prestados (Härmä, 2006). La tendencia en horarios laborales son los programas de trabajo alternativos, en los cuales hay un incremento en la duración de la jornada laboral, mientras que hay una reducción en el número de días laborados en la semana, es decir, existen en mayor número los turnos rotativos, nocturnos, mixtos y las jornadas de trabajo de más de 8 horas, conocidos como semana de trabajo comprimida (Paley et al., 1994).

Un horario de turno comprimido es una variación del horario de turno rotativo, donde un individuo trabaja cuatro días 10 o 12 horas antes de tomar 3 o 4 días libres. La idea es comprimir la cantidad de horas trabajadas en una pequeña cantidad de días para permitir más días libres (Cunningham, 1982). Como menciona Cunningham (1982), las semanas de trabajo comprimidas se han aceptado en el ambiente laboral, aunque sólo son aplicables en algunas organizaciones debido al tipo de trabajo que se realiza, ejemplo de ello son las organizaciones policiacas y de vigilancia, hospitales y en algunas maquiladoras donde el trabajo no exige un esfuerzo físico excesivo, tal es el caso de las empresas maquiladoras que se dedican sólo al ensamble.

Paley et al. (1994) mencionan que la semana de trabajo comprimida es una idea atractiva para muchas personas, porque se reduce el número de viajes de su casa al trabajo y viceversa, además de que hay menos tiempo de preparación, cierres de trabajo y aumento de 
los días de descanso. Åhsberg (1998), ha sugerido que en los turnos de semana comprimida se observa una mejora en la productividad, así como una reducción en los accidentes y errores, por el contrario, según Dawson y Fletcher (2001), las jornadas de 12 horas por 4 días a la semana producen más fatiga, un nivel bajo de sueño y actividad psicomotora que uno de 8 horas por cinco días a la semana.

Trabajar demasiadas horas, ya sea diarias o semanales, está directamente relacionado con un aumento en la tasa de accidentes y lesiones profesionales y la relación no depende de la edad, sexo, ocupación, o del sector de la industria. Ante el hecho de que la salud de los empleados se vea desfavorecida en torno a las largas jornadas laborales, se cuenta con el tema de la salud ocupacional, el cual es un tema de interés para los investigadores del área, puesto que se debe buscar un equilibrio entre las condiciones ligadas al trabajo y las condiciones biológicas de la persona, como menciona Härmä (2006).

Las condiciones sociales y materiales en que se realiza el trabajo pueden afectar el estado de bienestar de las personas en forma negativa. Los daños a la salud más evidentes y visibles son los accidentes del trabajo. De igual importancia son las enfermedades profesionales, aunque se sepa menos de ellas (Moretti, 2015).

En la fisiología y psique humana no ha sido posible identificar, de manera aislada o en ambas, si existe o emerge un mecanismo de retroalimentación específico de orden neuroendocrino capaz de detectar, procesar y hacer consciente a un individuo de la presencia de signos o señales para que tome un descanso, efectúe recesos o deba tener momentos de esparcimiento cuando desarrolla el trabajo. A falta de evidencia de este mecanismo en situaciones laborales donde no está establecido un límite de horas o se hace caso omiso de él, con frecuencia conduce al organismo a situaciones extremas que van más allá de la capacidad fisiológica del mismo para recuperarse (Vidacek \& Radosevic-Vidacek, 1994).

Es por ello que las consecuencias de las largas jornadas de trabajo en la salud del trabajador, han sido materia de estudio. Tal es el caso, del estudio realizado por Haro-García et al. (2007), donde demuestran que existe evidencia tanto empírica como teórica para decir que jornadas laborales muy extensas (12 horas o más) presentan invariablemente relación con disminución en el desempeño y eficiencia en el trabajo, errores en el cumplimiento de los sistemas de seguridad laboral, aumento de síntomas de fatiga, disminución del estado de alerta y acortamiento del periodo dedicado al sueño a cuatro horas o menos. Todas estas condiciones se asocian al aumento de factores de riesgo para sufrir patologías como el infarto agudo de miocardio y la aparición de síndrome metabólico.

Los turnos denominados semana de trabajo comprimida, forman parte de las condiciones laborales actuales y tienen un efecto directo sobre la salud de los empleados, principalmente en lo relacionado a la fatiga laboral. Además, de interferir directamente en los aspectos familiares, sociales y en otros factores de riesgo, tales como riesgos ergonómicos y de aspecto psicosocial. 


\section{Objetivo general}

Analizar y evaluar las jornadas de trabajo de 12 horas en las personas incorporadas a la industria manufacturera.

\section{Objetivos específicos}

- Identificar si las jornadas laborales de 12 horas ocasiona fatiga física y mental a los trabajadores con este tipo de turno.

- Determinar si las jornadas de trabajo de 12 horas están vinculadas con los factores de riesgo psicosocial.

\section{Materiales y métodos}

\section{Participantes}

En este estudio participaron 25 hombres y mujeres, de una empresa manufacturera de la región. Desarrollando actividades de ensamble, inspección y empaque de material. El personal pertenece al turno de semana de trabajo comprimida (12 horas), laborando de lunes a jueves de 7:00 AM a 7:00 PM. El contenido del puesto de trabajo, consiste en operaciones repetitivas, monótonas, bajo un estándar de producción que deben cumplir durante la jornada laboral.

El número de participantes no fue una muestra aleatoria, fueron los empleados adscritos a cierto departamento, que la empresa seleccionó para la toma de los datos de la presente investigación.

\section{Instrumentos}

Se aplicó el cuestionario subjetivo de fatiga de Yoshitake (1978) durante tres lapsos del periodo laboral: al iniciar la jornada de trabajo, a media jornada de trabajo y antes de finalizar la jornada de trabajo. El cuestionario está compuesto por tres dimensiones: dificultad de concentración, somnolencia o adormecimiento y fatiga física. Se aplica a la misma persona durante los tres lapsos de la jornada.

El siguiente instrumento utilizado fue la Norma Oficial Mexicana NOM-035-STPS2018 Factores de riesgo psicosocial en el trabajo-Identificación, análisis y prevención. El contenido del cuestionario abarca 5 categorías, 10 dominios y 25 dimensiones, siendo un total de 72 preguntas. La evaluación se aplicó en una ocasión durante el estudio. Cada encuestado accedió sin problema alguno, se realizó en su puesto específico de trabajo y cada uno fue evaluado conforme a la guía de referencia del instrumento. La duración del sondeo fue de 20 minutos aproximadamente.

Los resultados del cuestionario se califican de acuerdo a lo establecido en la tabla 1. 
Tabla 1. Tabla de calificación final guía de referencia III.

\begin{tabular}{|c|c|c|c|c|c|}
\hline $\begin{array}{c}\text { Resultado del } \\
\text { cuestionario }\end{array}$ & $\begin{array}{c}\text { Nulo o } \\
\text { despreciable }\end{array}$ & Bajo & Medio & Alto & Muy alto \\
\hline $\begin{array}{c}\text { Calificación } \\
\text { final del } \\
\text { cuestionario }\end{array}$ & Cfinal $<50$ & $50 \leq$ Cfinal $<75$ & $75 \leq$ Cfinal $<99$ & $99 \leq$ Cfinal $<140$ & Cfinal $>140$ \\
\hline
\end{tabular}

Para la evaluación de los riesgos ergonómicos, se seleccionó el método ART Tool (Health and Safety Executive [HSE], 2010), el cual está diseñado para evaluar las tareas repetitivas que implican el uso de las extremidades superiores, incluyendo parámetros para el análisis de jornadas laborales de más de 8 horas diarias y factores de riesgo psicosocial. El análisis consiste en analizar la tarea que realizan los operadores, para determinar si existe un nivel de riesgo significativo y de ser así, reducir ese nivel de riesgo en la tarea específica. La recolección de la información se realizó únicamente en una ocasión durante todo el estudio.

\section{Metodología}

Durante la aplicación de los cuestionarios, se puntualizó la importancia del estudio a los trabajadores, para obtener información relevante, la cual sería debidamente tratada bajos esquemas de privacidad de sus respuestas y datos. Los cuestionarios fueron aplicados individualmente en las estaciones de trabajo de cada participante. Una vez obtenidos los datos de la percepción de fatiga, se procedió al tratamiento estadístico, utilizando el método de análisis de varianza de medidas repetidas ANOVA (Triola, 2013, pp. 629). Además, la prueba de corrección de Bonferroni, para comparar la similitud entre la media de los factores (lapsos del turno). Se compararon las medias de cada uno de los factores, con un nivel de confianza $\alpha=0,05$.

Dado que $\mu 1=\mu 2=\mu 3$, se aplica la prueba de corrección de Bonferroni, donde se comparan las muestras intra-sujetos (comparaciones por parejas) entre los niveles del factor, es decir, se realiza una comparación entre cada uno de los denominados factores: inicio del turno, medio turno y fin del turno, de las muestras del día 1.

\section{Resultados y discusión}

\section{Fatiga}

Se presentan en la tabla 2, los resultados de las medias de los tres lapsos del turno en el que fue tomada la evaluación correspondiente al día 1, muestra 1, semana 1. 
Tabla 2. Estadísticos descriptivos de las medias de los lapsos del turno.

\begin{tabular}{|l|c|c|c|}
\hline Lapsos del turno & Media & Desviación estándar & N \\
\hline S1D1M1 & 6,60 & 9,866 & 25 \\
\hline S1D1M2 & 10,40 & 9,233 & 25 \\
\hline S1D1M3 & 24,00 & 16,330 & 25 \\
\hline
\end{tabular}

Nota. S1=semana 1, D1=día 1, M1=muestra 1, M2=muestra 2, M3=muestra 3.

Conociendo el nivel de significancia $\alpha=0,05$, se concluye que no se rechaza la hipótesis nula de medias iguales. No se rechaza la hipótesis nula, debido a que existe similitud de medias entre factores. Es decir, el factor inicio y el factor medio (lapsos del turno) cumplen esta característica. La siguiente figura muestra el intervalo entre las medias de cada factor, donde se aprecia la evolución de la sintomatología de fatiga con base a la encuesta realizada a los trabajadores, correspondiente al día laboral.

Figura 1. Gráfica de intervalo entre las medias de los lapsos del turno.

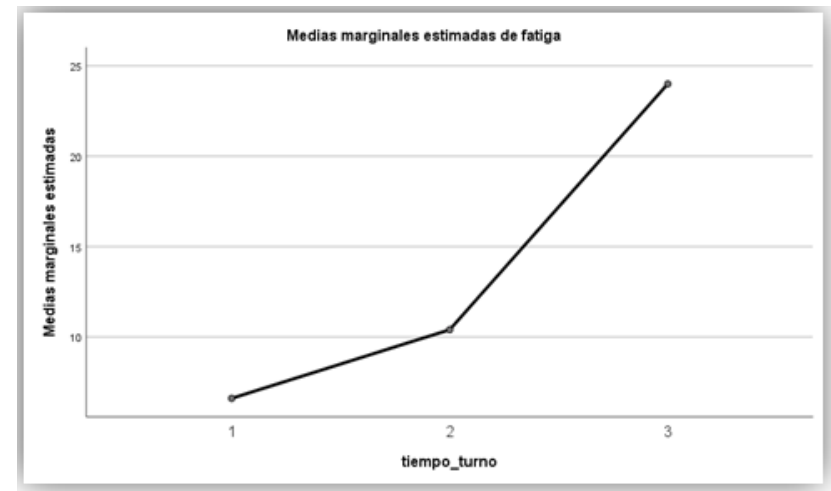

La siguiente figura muestra los datos de una semana de trabajo, donde se pueden observar los intervalos de los valores de las medias entre los factores del turno de inicio, medio y fin (lapsos de tiempo) en los que se toma la muestra. Se aprecia la evolución del índice de queja de fatiga entre cada factor y se nota visiblemente un incremento desde el inicio del turno hasta el fin del mismo, durante una semana de trabajo. 
Figura 2. Gráfica de intervalo entre las medias de los lapsos del turno de una semana de trabajo.

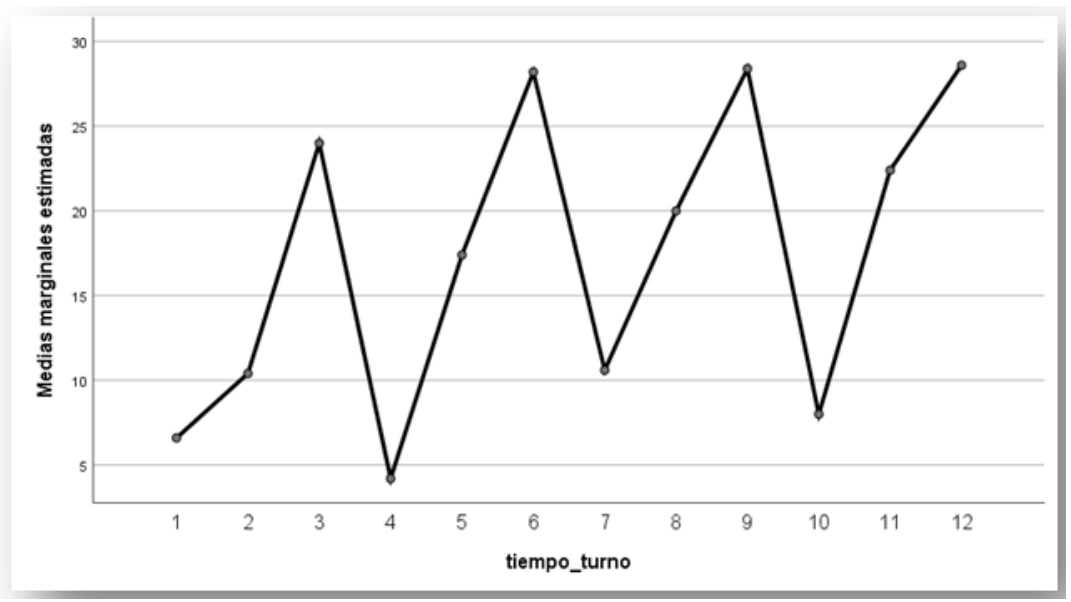

De la información obtenida de la semana de trabajo, se puede concretar qué factores son los que comparten similitud entre sus medias, lo cual indica que, al inicio del turno y en el tiempo transcurrido al medio turno, los datos de los resultados del cuestionario de fatiga de Yoshitake son similares y no se aprecia una "insatisfacción" por parte del trabajador, a diferencia del tiempo que transcurre del medio turno al tiempo antes de finalizar el mismo, donde el incremento del factor de queja de fatiga se nota en gran medida, con respecto a la información proporcionada en las figuras anteriores. Lo mismo sucede en el intervalo entre las medias de los lapsos del turno del mes completo de trabajo, donde se aprecia en la figura 3, el índice de queja de fatiga entre el lapso del medio turno y antes de finalizar el turno.

Figura 3. Gráfica de intervalo entre las medias de los lapsos del turno del mes completo de trabajo.

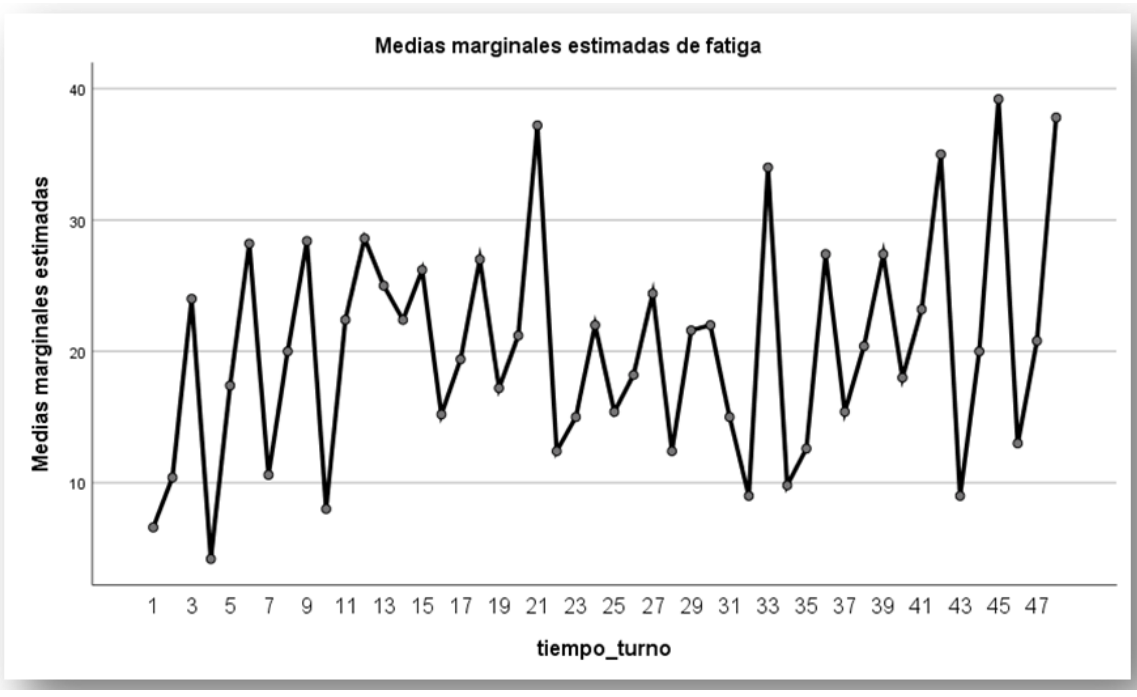

Factores de riesgo psicosocial

La información obtenida mediante la guía de referencia de la Norma Oficial Mexicana NOM035-STPS-2018, arrojó los resultados que se observan en la figura 4. 
Figura 4. Gráfica de calificación final de los resultados de los FRPS.

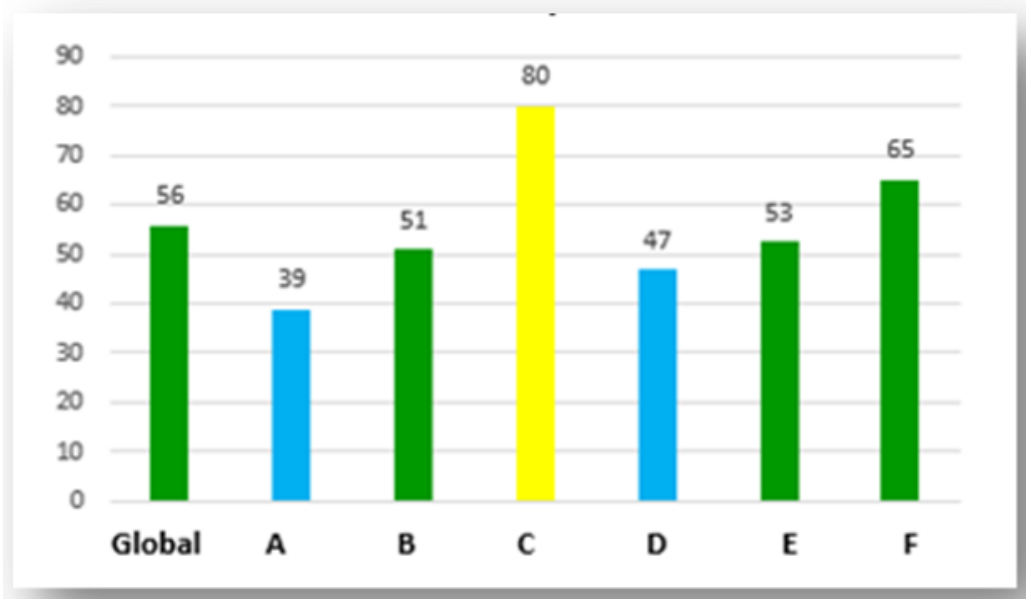

El análisis indica que en las líneas de producción A y D existe un nivel de riesgo nulo; en las líneas B, E, F existe un nivel de riesgo bajo, es decir, sí se pueden encontrar algunas dimensiones correspondientes a los factores de riesgo psicosocial. Finalmente, la línea $\mathrm{C}$ se encuentra en un nivel de riesgo medio.

De tal modo, se tiene que la categoría denominada "factores propios de la actividad" y “organización del tiempo de trabajo" están dentro de los niveles de riesgo medio, en las líneas donde se aplicó el cuestionario, tal como se observa en la figura 5.

Figura 5. Gráfica de calificación por categoría de resultados de FRPS.

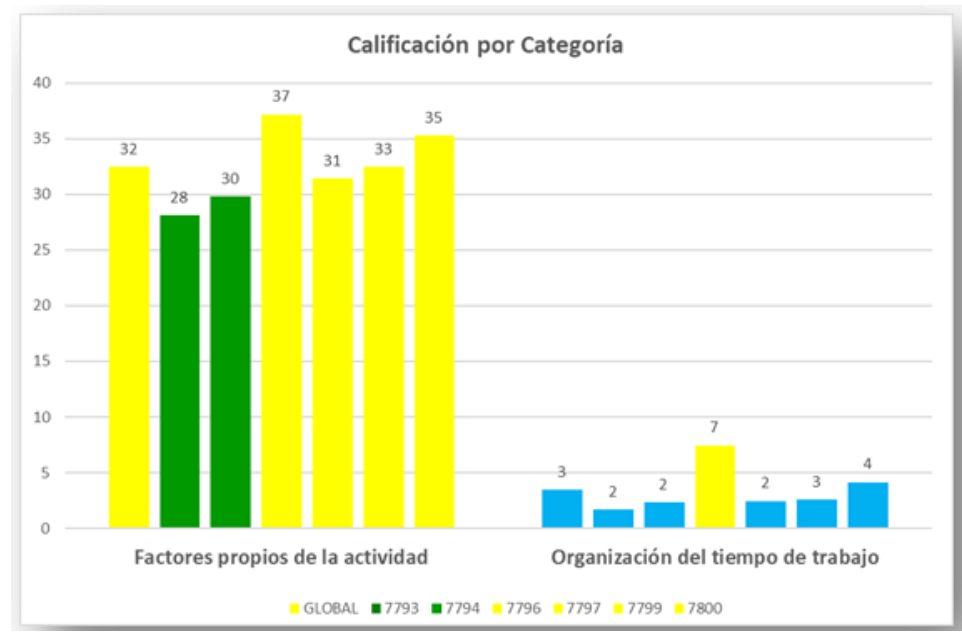

Factores de riesgo ergonómico

Los resultados obtenidos con la aplicación de la herramienta ART Tool deja en claro que las actividades realizadas por los operadores, a simple vista, pueden observarse como seguras, pero los resultados muestran que se requiere un ajuste meticuloso para mejorar las estaciones de trabajo. Se encontró que el 12,07\% de los puestos de trabajo tienen un nivel de riesgo bajo, el 24,14\% corresponde a un nivel de riesgo medio y el 63,78\% corresponde a un nivel de riesgo alto. 


\section{Conclusiones}

Los resultados obtenidos en la presente investigación muestran que la fatiga está presente en este tipo de esquema laboral, llamado semana de trabajo comprimida.

El análisis de este estudio justifica el objetivo planteado, referente a la existencia e incremento de fatiga laboral en las personas adscritas a este tipo de turno. La evidencia estadística muestra un incremento de fatiga entre los factores (medio turno y fin del turno).

La presencia de fatiga percibida por los trabajadores fue notoria, ya que las actividades que realizan carecen del uso de sus habilidades, del control de las tareas, así como la toma de decisiones; factores que influyen en la carga mecánica y demandas físicas propias de operaciones repetitivas. Los trabajos repetitivos y monótonos acumulan mucha más tensión, tal como se señala en estudios incluidos en esta investigación.

En el desarrollo de la investigación, se observó que uno de los mayores retos de la ergonomía ha sido el estudio de la interacción del hombre frente a los requerimientos físicos, tales como, posturas, fuerza y movimiento. Cuando estos requerimientos sobrepasan la capacidad de respuesta del individuo no hay una adecuada recuperación biológica, este esfuerzo puede asociarse con la presencia de dolencias, lesiones o estrés, todas ellas relacionadas con el trabajo. Existe una estrecha relación con posibles dolencias musculoesqueléticas, el diseño del sistema, el puesto de trabajo y, finalmente, la carga mental asociada a la actividad efectuada.

El organismo humano, el trabajador, se debe considerar como una totalidad o sistema biopsicosocial. Donde los elementos externos afectan la totalidad del sistema. Es decir, esos efectos resaltan en los factores psicosociales y, si aumentan, también se incrementan los riesgos ergonómicos, de seguridad e higiene. De la misma manera, si se incrementan los riesgos antes mencionados, también se desarrollan riesgos psicosociales, originando conductas alteradas o precipitadas que originan errores, accidentes de trabajo y una mayor vulnerabilidad en la persona.

Es recomendable continuar con las evaluaciones de los factores de riesgo para futuras intervenciones, sobre todo para erradicar o minimizar este tipo de riesgo al que están expuestos los trabajadores. 


\section{Referencias}

Åhsberg, E. (1998). Perceived fatigue related to work. National Institute for Working Life. https://citeseerx.ist.psu.edu/viewdoc/download?doi=10.1.1.460.228\&rep=rep1\&type=pdf

Cunningham, J. B. (1982). Compressed shift schedules: Altering the relationship between work and non-work. Public Administration Review, 42(5), 438. https://doi.org/10.2307/975646

Dawson, D., \& Fletcher, A. (2001). A quantitative model of work-related fatigue: Background and definition. Ergonomics, 44(2), 144-163. https://doi.org/10.1080/00140130119399

Härmä, M. (2006). Norwegian National Institute of Occupational Health in relation and health to work workhours stress, recovery. Scandinavian Journal of Work, Environment \& Health, 32(6), 502-514. http://www.jstor.org/stable/40967602

Haro-García, L., Sánchez-Román, R., Juárez-Pérez, C. A., \& Larios-Díaz, E. (2007). Justificaciones médicas de la jornada laboral maxima de ocho horas. Revista Médica del Instituto Mexicano del Seguro Social, 45(2), 191-197. https://www.medigraphic.com/cgibin/new/resumen.cgi?IDARTICULO $=11764$

Health and Safety Executive (2010). Assessment of repetitive tasks of the upper limbs (the ART tool). Guidance for employers. http://www.hse.gov.uk/pubns/indg438.pdf

Moretti, C. A. (2015). Duración de la jornada laboral: Implicancias sanitarias y políticoeconómicas. Revista Chilena de Terapia Ocupacional, 15(1), 57-64. https://doi.org/10.5354/0719-5346.2015.37130

Norma Oficial Mexicana NOM-035-STPS-2018. Factores de riesgo psicosocial en el trabajoIdentificación, análisis y prevención. Diario Oficial de la Federación, Ciudad de México, México, 23 de octubre de 2018.

Paley, M. J., Herbert, L. B., \& Tepas, D. I. (1994). Long term evaluation of a compressed, rapidly rotating work schedule. Proceedings of the Human Factors and Ergonomics Society Annual Meeting, 38(12), 749-753. https://doi.org/10.1177/154193129403801207

Triola, M. F. (2013). Estadística. Pearson Educación.

Vidacek, S., \& Radosevic-Vidacek, B. (1994). Chronobiological features of the 12-hour working day. Arhiv Za Higïjenu Rada i Toksikologïju, 45(4), 379-387. https://hrcak.srce.hr/145857

Villegas, J., Noriega, M., Martínez, S., \& Martínez, S. (1997). Trabajo y salud en la industria maquiladora mexicana: Una tendencia dominante en el neoliberalismo dominado. Cadernos de Saude Publica, 13(Suppl 2), 123-134. https://doi.org/10.1590/So102311X1997000600011

Yoshitake, H. (1978). Three characteristic patterns of subjective fatigue symptoms. Ergonomics, 21(3), 231-233. https://doi.org/10.1080/00140137808931718 